\title{
Article \\ Bacillus subtilis ANSB168 Producing D-alanyl-D-alanine Carboxypeptidase Could Alleviate the Immune Injury and Inflammation Induced by Ochratoxin A
}

\author{
Hanrui Qing ${ }^{\dagger}$, Xueting Huo ${ }^{\dagger}$, Shimeng Huang, Lihong Zhao, Jianyun Zhang, Cheng Ji and Qiugang Ma *(D) \\ State Key Laboratory of Animal Nutrition, College of Animal Science and Technology, China Agricultural \\ University, Haidian District, Beijing 100094, China; s20193040584@cau.edu.cn (H.Q.); huoxt@lamic.com.cn (X.H.); \\ shimengh@hotmail.com (S.H.); lihongzhao@126.com (L.Z.); jyzhang@cau.edu.cn (J.Z.); jcanl@cau.edu.cn (C.J.) \\ * Correspondence: maqiugang@cau.edu.cn; Tel.: +86-10-6273-2774 \\ † These authors contributed equally to this study.
}

check for updates

Citation: Qing, H.; Huo, X.; Huang, S.; Zhao, L.; Zhang, J.; Ji, C.; Ma, Q. Bacillus subtilis ANSB168 Producing D-alanyl-D-alanine Carboxypeptidase Could Alleviate the Immune Injury and Inflammation Induced by Ochratoxin A. Int. J. Mol. Sci. 2021, 22, 12059. https://doi.org/10.3390/ ijms222112059

Academic Editor: Yuseok Moon

Received: 27 September 2021 Accepted: 5 November 2021 Published: 8 November 2021

Publisher's Note: MDPI stays neutral with regard to jurisdictional claims in published maps and institutional affiliations.

Copyright: (c) 2021 by the authors. Licensee MDPI, Basel, Switzerland. This article is an open access article distributed under the terms and conditions of the Creative Commons Attribution (CC BY) license (https:// creativecommons.org/licenses/by/ $4.0 /)$

\begin{abstract}
Ochratoxin A (OTA) is toxic to animals and threatens food safety through residues in animal tissues. A novel degrading strain Bacillus subtilis ANSB168 was isolated and further investigated. We cloned D-alanyl-D-alanine carboxypeptidase DacA and DacB from ANSB168 and over-expressed them in Escherichia coli Rosetta (DE3). Then, we characterized the OTA degradation mechanism of DacA and DacB, which was degrading OTA into OT $\alpha$. A total of 45 laying hens were divided into three equal groups. The control group was fed basal feed, and other groups were administered with OTA $\left(250 \mu \mathrm{g} / \mathrm{kg}\right.$ of feed). A freeze-dried culture powder of ANSB168 $\left(3 \times 10^{7} \mathrm{CFU} / \mathrm{g}, 2 \mathrm{~kg} / \mathrm{T}\right.$ of feed) was added to one of the OTA-fed groups for 28 days from day one of the experiment. We found that OTA significantly damaged the kidney and liver, inducing inflammation and activating the humoral immune system, causing oxidative stress in the layers. The ANSB168 bioproduct was able to alleviate OTA-induced kidney and liver damage, relieving OTA-induced inflammation and oxidative stress. Overall, DacA and DacB derived from ANSB168 degraded OTA into OT $\alpha$, while the ANSB168 bioproduct was able to alleviate damages induced by OTA in laying hens.
\end{abstract}

Keywords: ochratoxin A; mycotoxin; degradation; carboxypeptidase; poultry

\section{Introduction}

Ochratoxin A (OTA), produced by several strains of Aspergillus and Penicillium [1-6], is a naturally occurring mycotoxin that commonly contaminates foods and feeds, causing enormous harm to humans and animals. Notably, OTA is defined as a group 2B carcinogen to humans by the International Agency for Research on Cancer (IARC, 1993). According to the European Commission recommendation 2006/576/EC, the maximum tolerable concentration of OTA is $100 \mu \mathrm{g} / \mathrm{kg}$ in poultry's complete feed. The target organs of OTA are considered to be the kidney and liver. Studies have reported that OTA causes nephrotoxicity, hepatotoxicity, immunotoxicity, teratogenicity, genotoxicity, and carcinogenicity in animals [7-10]. Plenty of animal feeding trials have been performed to evaluate the potential toxicity of OTA. Injury to kidneys of OTA-treated rats was observed in histological tests, which related to OTA-induced oxidative stress [11]. Immune response and antioxidant defense were affected in the gut and kidney of piglets, owing to long time exposure at a low dose of OTA [12]. Likewise, hepatocyte necrosis was observed in piglets after feeding them OTA-contaminated feed for 33 days [13]. OTA causing oxidative stress and immunosuppression in broiler chicks was also reported, including a significant dose-dependent decrease in superoxide dismutase (SOD), glutathione peroxidase (GSHPx), total antioxidant status (TAS), immunoglobulin and total antibody titers, phagocytic potential, and lymphoproliferative responses [14,15].

OTA is thermally stable up to $18^{\circ} \mathrm{C}$ [16], which means most ochratoxins in agriculture products can pass through industrial processing programs. Consequently, this contributes 
to the prevalence of mycotoxin contamination, causing enormous economic losses and threatening human health.

Different protocols that are categorized into physical, chemical, and biological strategies have been developed to control levels of OTA in foods and feeds and prevent the toxic effects of OTA in animals and humans [17]. For example, with $3 \mathrm{~h}$ of UV irradiation, $81-86 \%$ of OTA in feed can be degraded [18], but it requires extra equipment and is time-consuming. Chemical agents, such as ozone, ammonium, bisulfites, and alkaline hydrolysis, are commonly applied in detoxification $[15,19]$. However, its effectiveness varies and raises problems of chemical residues. Biotechnological strategies are widely used in eliminating OTA because it is environmentally friendly and effective. Several biocontrol strains like Burkholderia cepacia, Brevibacterium casei, Aspergillus niger, and Alcaligenes faecalis were researched and evaluated with varying degrees of success [20-24]. The direct degradation of OTA to a less toxic substance ochratoxin $\alpha(\mathrm{OT} \alpha)$ is the most significant mechanism [22], but some microorganisms can also have antifungal properties and inhibit OTA production $[25,26]$.

The chemical structure of OTA is 7-carboxyl-5-chloro-8-hydroxyl-3,4-dihydro-3Rmethyl-isocoumarin-7-L- $\beta$-phenylalanine, which is a phenylalanyl derivative of a dihydroisocoumarin. Between the dihydroisocoumarin moiety and the L- $\beta$-phenylalanine, there is an amide bond linkage $[27,28]$. The enzyme carboxypeptidase can catalyze the hydrolysis of OTA at the amide bond into less toxic OT $\alpha$ and non-toxic L- $\beta$-phenylalanine (Phe) [29]. Much of the research in OTA detoxification has examined the effectiveness of a variety of carboxypeptidases. Among them, carboxypeptidases derived from bovine or porcine sources are the most investigated, such as bovine pancreas carboxypeptidase $\mathrm{A}$ (CPA, 3.4.17.1) expressed without propeptide and signal peptide [30], bovine pancreas CPA immobilized with a zeolitic imidazolate framework [31], and porcine carboxypeptidase B (CPB, 3.4.17.2) [32]. However, compared with high-cost animal sources, carboxypeptidases from microbes are a more promising prospect. Researchers have studied carboxypeptidases from Bacillus amyloliquefaciens ASAG1 [33], Bacillus subtilis CW14 [34], and Lysobacter sp. CW239 [35]. Although a number of articles have reported different kinds of carboxypeptidases derived from different OTA-degrading strains, the mechanism and purified enzyme of a novel screened strain Bacillus subtilis have yet to be researched.

The present study aimed to investigate the detoxification effectiveness and mechanism of Bacillus subtilis ANSB168 in vivo and in vitro. The strain ANSB168 in this study was isolated from the cecum of a donkey and could degrade $47.0 \%$ of OTA and produce OT $\alpha$ within $18 \mathrm{~h}$ in vitro, which led to a hypothesis that ANSB168 has the biological activity of D-alanyl-D-alanine carboxypeptidase. In this study, D-Ala-D-Ala carboxypeptidase DacA and DacB from ANSB168 were cloned and over-expressed in Escherichia coli Rosetta (DE3). Moreover, OTA degradation kinetics and the mechanism of recombinant DacA and DacB were characterized. Furthermore, an animal trial was conducted to evaluate the ameliorative effects of the freeze-dried culture of ANSB168 in OTA-fed poultry. The results obtained from this study demonstrate that the strain ANSB168 and its specific enzyme could be applied in OTA degradation.

\section{Results}

\subsection{Isolation and Identity of OTA-Degrading Bacteria ANSB168}

Strain ANSB168 was isolated by the enrichment method from the cecum of donkeys, and the strain showed efficient degradation activity of OTA. The isolated strain ANSB168 is a gram-positive bacillus strain (Figure 1A). The almost complete 16S rRNA gene sequence (1427 bp) of ANSB168 was cloned and analyzed. The constructed phylogenetic tree showed that ANSB168 was a member of the genus Bacillus and was in a separate phylogenetic clade with Bacillus subtilis (Figure 1B). The $16 \mathrm{~S}$ rDNA sequence had been uploaded to the National Center for Biotechnology Information (NCBI) GenBank and was obtained with the accession number OK663194. The degradation tests showed that ANSB168 was able to 
degrade $47.0 \%$ of OTA and produce OT $\alpha$ within $18 \mathrm{~h}$ (Figure 2), indicating that ANSB168 was capable of breaking the amino bond of OTA.

A

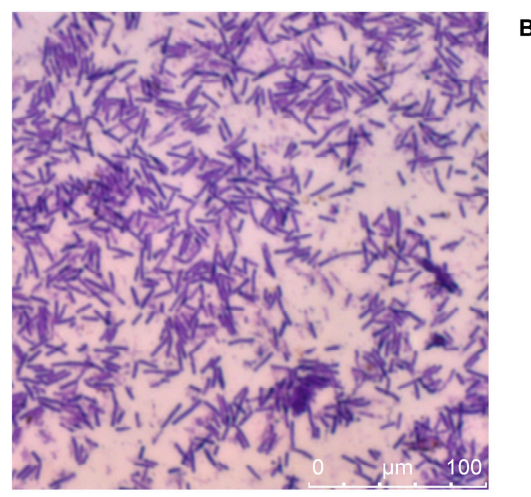

B

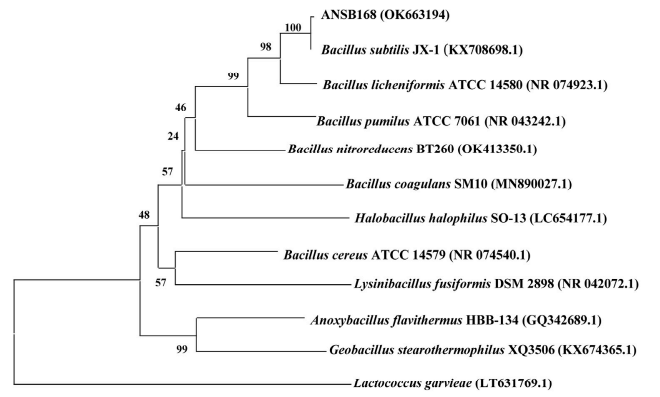

0.2020

Figure 1. Gram staining and phylogenetic of strain ANSB168. (A) Gram staining of ANSB168 (×630). (B) Neighbor-joining phylogenetic tree based on 16S rRNA gene sequences, showing the phylogeny position of ANSB168 in genus Bacillus. Bootstrap percentages of 1000 replicates are given at branch points, and GenBank accession numbers are given in parentheses. Bar, 1\% nucleotide substitutions per 100 nt.
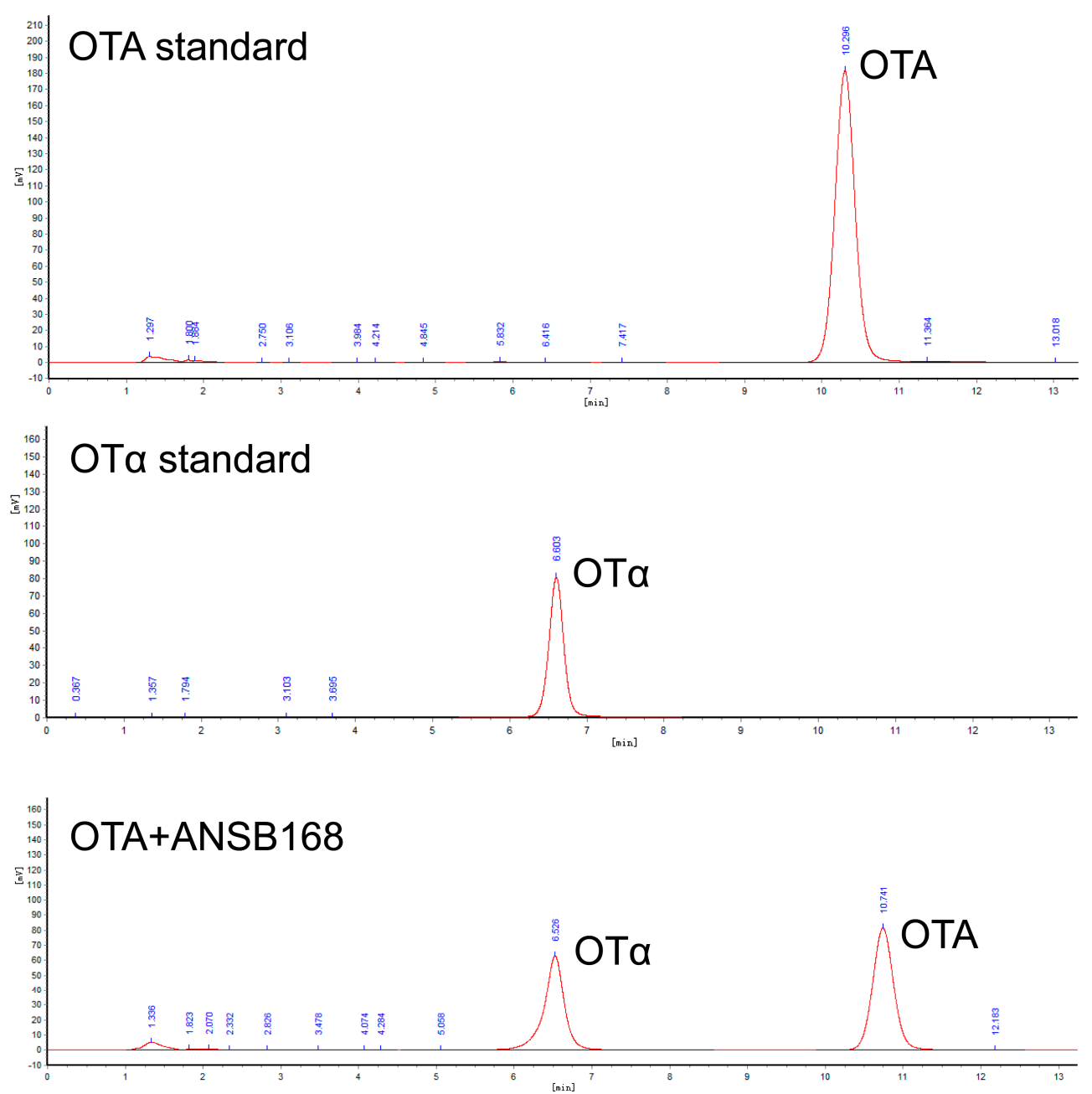

Figure 2. OTA degradation of strain ANSB168, including the High-performance liquid chromatography (HPLC) of OTA standard, OT $\alpha$ standard, and 18 h OTA incubation with ANSB168. 


\subsection{Cloning and Expression of DacA and DacB in E. coli}

The D-Ala-D-Ala carboxypeptidase DacA and DacB genes were amplified by PCR according to the genomic sequence of ANSB168. sodium dodecyl sulfate-polyacrylamide gel electrophoresis (SDS-PAGE) analysis confirmed the purity of the recombinant DacA with a gene sequence of $1239 \mathrm{bp}$ and $\mathrm{DacB}$ with a gene sequence of $1068 \mathrm{bp}$ (Figure 3A). The multiple alignments of the DacA and DacB amino acid sequences were highly homologous to carboxypeptidases. The PCR obtained DNA fragment was purified, digested with NdeI and $\mathrm{XhoI}$, and then ligated into expression vector pET-31b (Figure 3B). The recombinant plasmid pET-31b-DacA/pET-31b-DacB was transformed into E. coli Rosseta (DE3), followed by induction with $0.4 \mathrm{mM}$ isopropyl-b-D-thiogalactoside (IPTG) at $37^{\circ} \mathrm{C}$ for $3 \mathrm{~h}$. As shown in Figure 3C, E. coli Rosseta (DE3)-pET-31b-DacA and E. coli Rosseta (DE3)-pET-31b-DacB proteins were efficiently expressed in the supernatant as well as in the precipitant. The expressed recombinant (DacA and DacB) was purified through Ni-NTA column affinity chromatography. SDS-PAGE analysis confirmed the purity of the recombinant DacA with a molecular weight of $46 \mathrm{kDa}$ and $\mathrm{DacB}$ with a molecular weight of $41 \mathrm{kDa}$ (Figure 3D). Moreover, purified DacA and DacB were recognized by the mouse anti-His tag antibody by Western blot analysis (Figure $3 \mathrm{E}$ ). The expression quantities of DacA and DacB were $10.26 \mathrm{mg} / \mathrm{L}$ and $9.24 \mathrm{mg} / \mathrm{L}$, respectively.

A

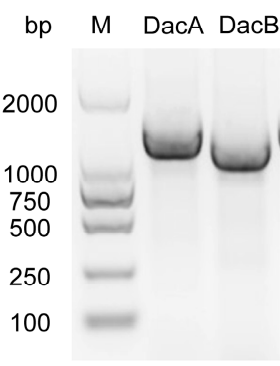

D

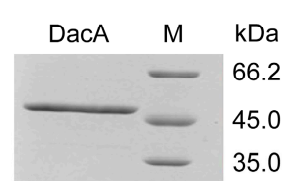

B
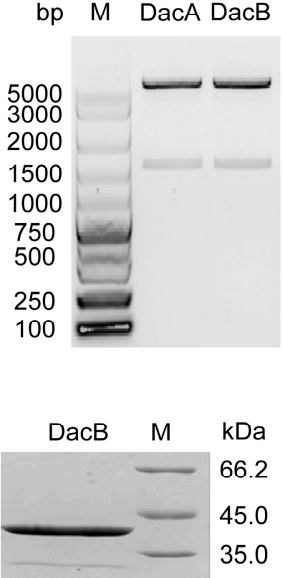

C

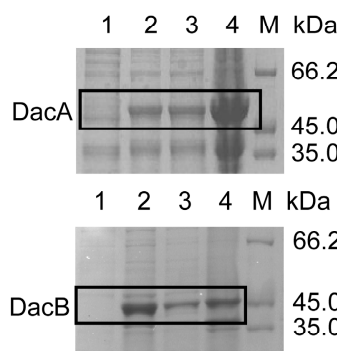

E

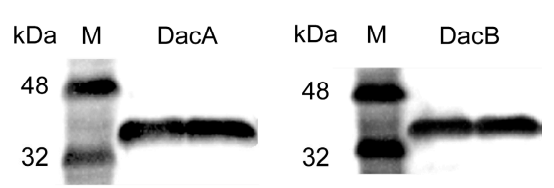

Figure 3. Gene cloning, overexpression, and protein purification of D-Ala-D-Ala carboxypeptidase DacA and DacB. (A) Agarose gel electrophoresis of PCR products of gene DacA and DacB. (B) Agarose gel electrophoresis of PCR products of gene DacA and DacB from recombinant plasmid. (C) SDSPAGE analysis showing overexpression of the recombinant DacA and DacB in E. coli DE3: lane 1, disrupted recombinant cells without IPTG; lane 2, disrupted recombinant cells with IPTG; lane 3, disrupted recombinant cells with IPTG (supernatant); lane 4, disrupted recombinant cells with IPTG (precipitant). (D) SDS-PAGE analysis showing the purified DacA and DacB. (E) Western blot analysis of recombinant DacA and DacB.

On the whole, the DacA gene was $1239 \mathrm{bp}$ in length, encoding 412 amino acids, of which its theoretical molecular weight was $45.3 \mathrm{kDa}$, while the DacB gene was $1068 \mathrm{bp}$ in length, encoding 355 amino acids, and its theoretical molecular weight was $40.1 \mathrm{kDa}$. The expressed DacA/DacB protein had a histidine tag $(6 \times$ His $)$ at the C-terminus. Therefore, the molecular weight displayed on the protein gel chart is slightly larger than that of the prediction.

\subsection{D-alanyl-D-alanine Carboxypeptidase DacA and DacB Degradation Activity and Biochemical Characteristics}

Various buffers at different $\mathrm{pH}$ values were used to investigate the optimum $\mathrm{pH}$ of the purified recombinant DacA and DacB. The optimum $\mathrm{pH}$ values for OTA degradation of DacA and DacB were 7.0 and 7.5, respectively (Figure 4A). Notably, DacA exhibited higher 
relative degradation activity than $\mathrm{DacB}$ when evaluated in $\mathrm{pH} 5-7$, while there were no OTA degradation activities of either enzyme detected at $\mathrm{pH}$ below 5.0. The degradation efficiency of DacA was above $35 \%$ within $72 \mathrm{~h}$ when the $\mathrm{pH}$ was between 6.5 and 7.5 . When the $\mathrm{pH}$ was above 7.5 , the activity of DacB to degrade OTA decreased rapidly.

A

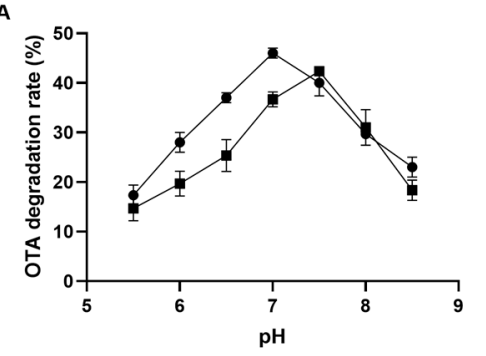

C

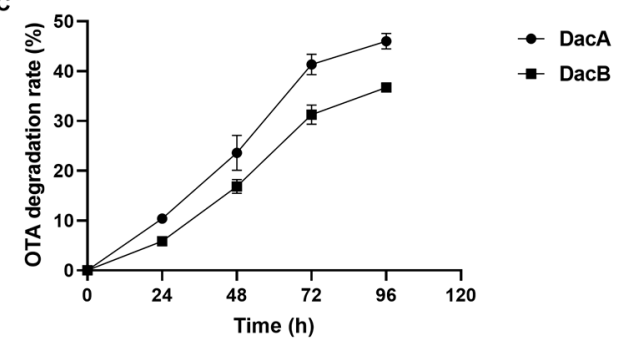

B

- DacA

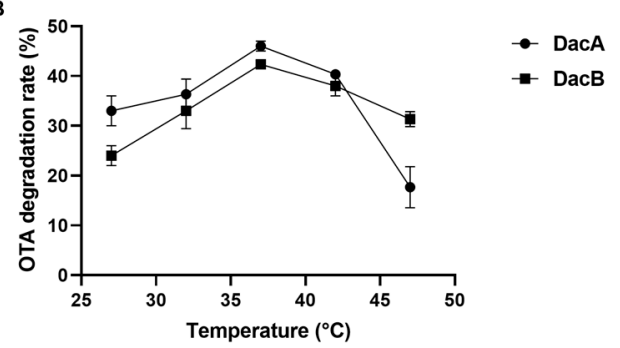

Figure 4. Detoxification characteristics of OTA by DacA and DacB: (A) the optimum pH of DacA and $\mathrm{DacB} ;(\mathbf{B})$ the optimum temperature of DacA and DacB; $(\mathbf{C})$ the degradation time of OTA by DacA and DacB.

The optimum $\mathrm{pH}$ was used to evaluate the preferred temperature of DacA and DacB. The optimal temperature for OTA degradation was found to be $37{ }^{\circ} \mathrm{C}$ for both DacA and DacB (Figure 4B). When the reaction temperature was higher than $42{ }^{\circ} \mathrm{C}$, the OTA degradation activity of DacA decreased significantly. In the range of $32-47^{\circ} \mathrm{C}$, DacB maintained a relatively high OTA degradation activity; the OTA degradation efficiency was higher than $30 \%$ after $72 \mathrm{~h}$ of incubation.

The kinetic parameters, $\mathrm{Km}$ and Vmax, were $2.74 \mu \mathrm{g} / \mathrm{mL}$ and $73.53 \mathrm{ng} / \mathrm{h} / \mathrm{mg}$ for DacA and $1.14 \mu \mathrm{g} / \mathrm{mL}$, and $42.74 \mathrm{ng} / \mathrm{h} / \mathrm{mg}$ for DacB when determined at $37^{\circ} \mathrm{C}$ and optimal $\mathrm{pH}$. The OTA degradation ratio increased over the incubation time. DacA and DacB were able to degrade $45 \%$ and $42 \%$ of OTA after $72 \mathrm{~h}$, respectively (Figure $4 \mathrm{C}$ ).

\subsection{Degraded Product Identification of DacA and DacB}

High-performance liquid chromatography (HPLC) analysis indicated that the DacA and $\mathrm{DacB}$ degradation products were eluted as a peak with a retention time of $6.7 \mathrm{~min}$ that had the same transition time of OT $\alpha$ (Figure 5), suggesting that DacA and DacB break the amide bond of OTA. These results demonstrate that DacA and DacB possess OTAdegrading activity, and the strain ANSB168 can eliminate OTA employing biodegradation. 

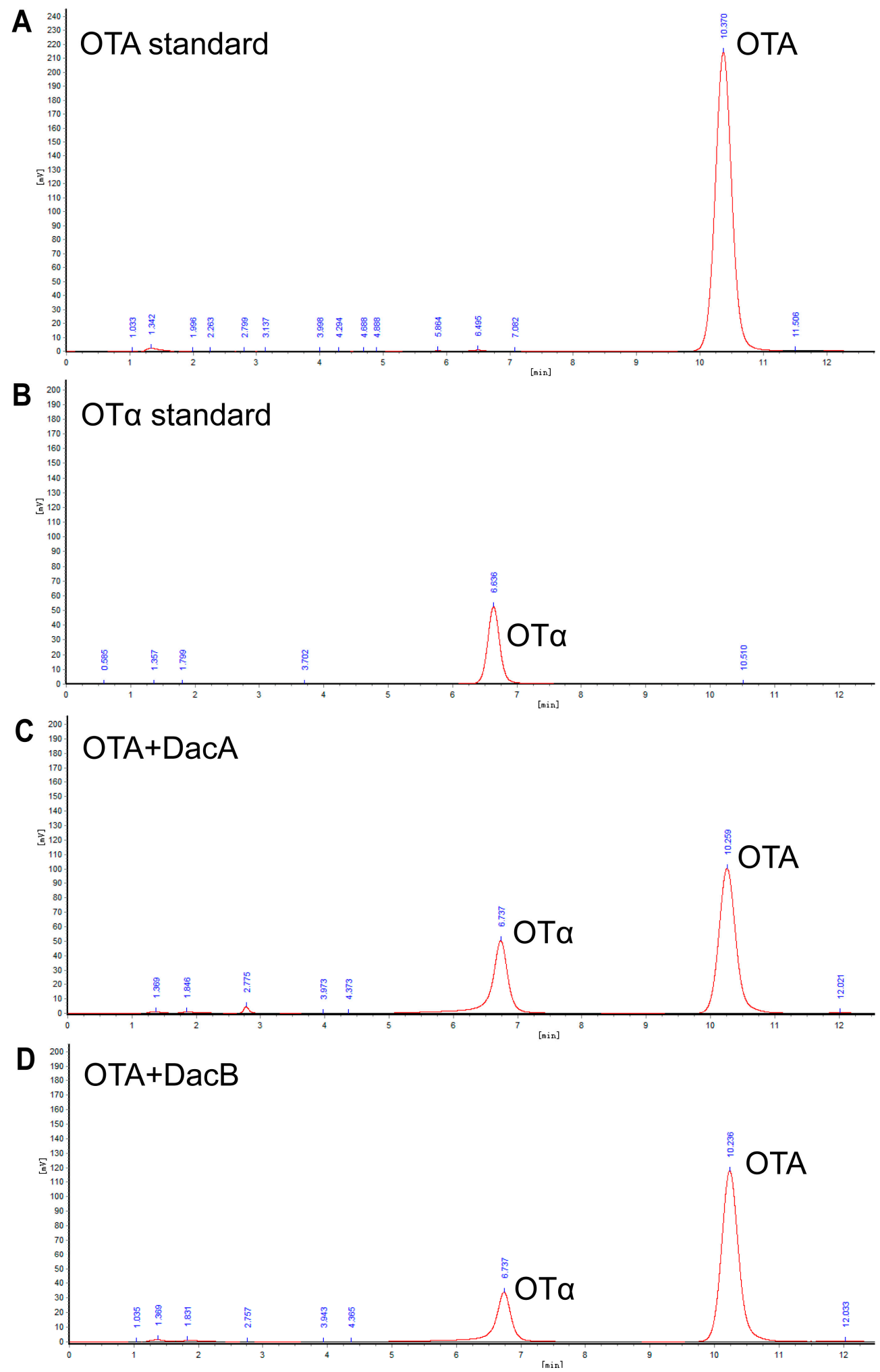

Figure 5. Determination of OTA and OT $\alpha$ by HPLC: (A) OTA standard; (B) OT $\alpha$ standard; (C) OTA standard processed by DacA; and (D) OTA standard processed by DacB.

2.5. Ameliorative Effects of Bacillus subtilis ANSB168 in Laying Hens 2.5.1. Production Performance

Feeding layers with OTA at the concentration of $250 \mu \mathrm{g} / \mathrm{kg}$ had negative effects on laying hens' measured performance parameters (i.e., egg production ratio, average egg weight, daily egg production, feed/egg ratio, and average daily feed intake). In Figure 6A, 
the daily feed intake of the OTA-fed group and the OTA+ANSB168 group was significantly decreased compared with the control group $(p<0.01)$. Both the egg production ratio (Figure $6 \mathrm{~B}, p=0.059$ ) and the daily egg production (Figure $6 \mathrm{C}, p=0.099$ ) of the OTA-fed group had a downward trend compared with the control. When compared with the control group, the egg production ratio and the daily egg production decreased $5.49 \%$ and $6.25 \%$, respectively. Although not statistically significant due to the variable differences within groups, the OTA-fed group's feed/egg ratio increased by $5.28 \%$ (Figure $6 \mathrm{D}$ ) and average egg weight decreased $0.71 \%$ (Figure $6 \mathrm{E}$ ) compared with the control group. However, the OTA+ANSB168 treatment group showed ameliorative effects on feed consumption, egg production ratio, egg production, feed/egg ratio, and egg weight compared with the OTA-fed group (Figure 6B-E).

A

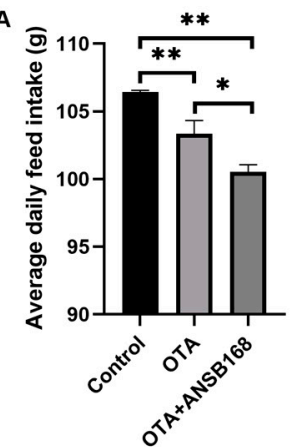

D

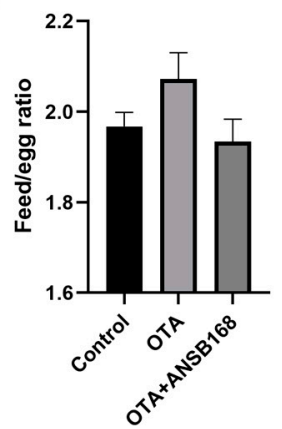

B

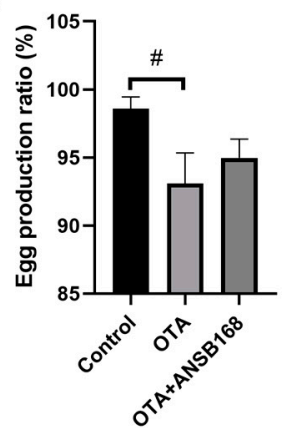

E

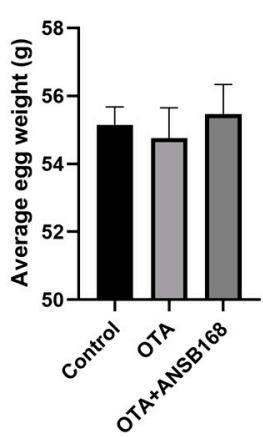

C

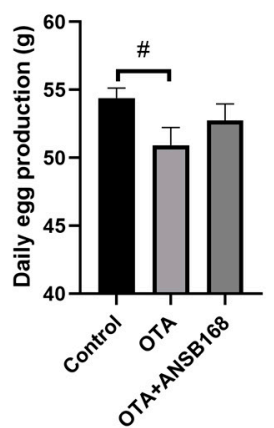

Figure 6. Production performances of layers during the study ( $n=15$, mean \pm SEM). (A) Effects of OTA and ANSB168 on layers' average daily feed intake (g). (B) Effects of OTA and ANSB168 on layers' egg production ratio (\%). (C) Effects of OTA and ANSB168 on layers' daily egg production (g). (D) Effects of OTA and ANSB168 on layers' feed/egg ratio. (E) Effects of OTA and ANSB168 on layers' average egg weight (g). Data were analyzed with ANOVA and Tukey's HSD and significant differences were defined as \# $0.05 \leq p<0.10,{ }^{*} p<0.05$, and ${ }^{* *} p<0.01$.

\subsubsection{Serum Biochemical Parameters}

The levels of the serum biochemical parameters for the layers kept on different dietary treatments for 28 days have been presented in Figure 7. The levels of the kidney damage parameters alanine aminopeptidase (AAP) $(p<0.01)$, leucine aminopeptidase (LAP) $(p<0.05)$, and phosphoenolpyruvate carboxykinase (PEPCK) $(p<0.05)$ significantly increased in the OTA-fed group compared with the control group. The level of creatinine $(\mathrm{Cr})$ in the OTA-fed group was higher than that of the control group, though it was not statistically significant. As for liver damage parameters, including alkaline phosphatase (ALP), aspartate aminotransferase (AST), and alanine aminotransferase (ALT), there were no statistically significant differences between the OTA and the control group. However, numerically worse ALP and AST could be observed in the OTA group compared with the control. The results elucidate that $250 \mu \mathrm{g} / \mathrm{kg}$ of OTA in feed induced certain kidney and liver damages, and the injury in the kidney was worse than in the liver. 

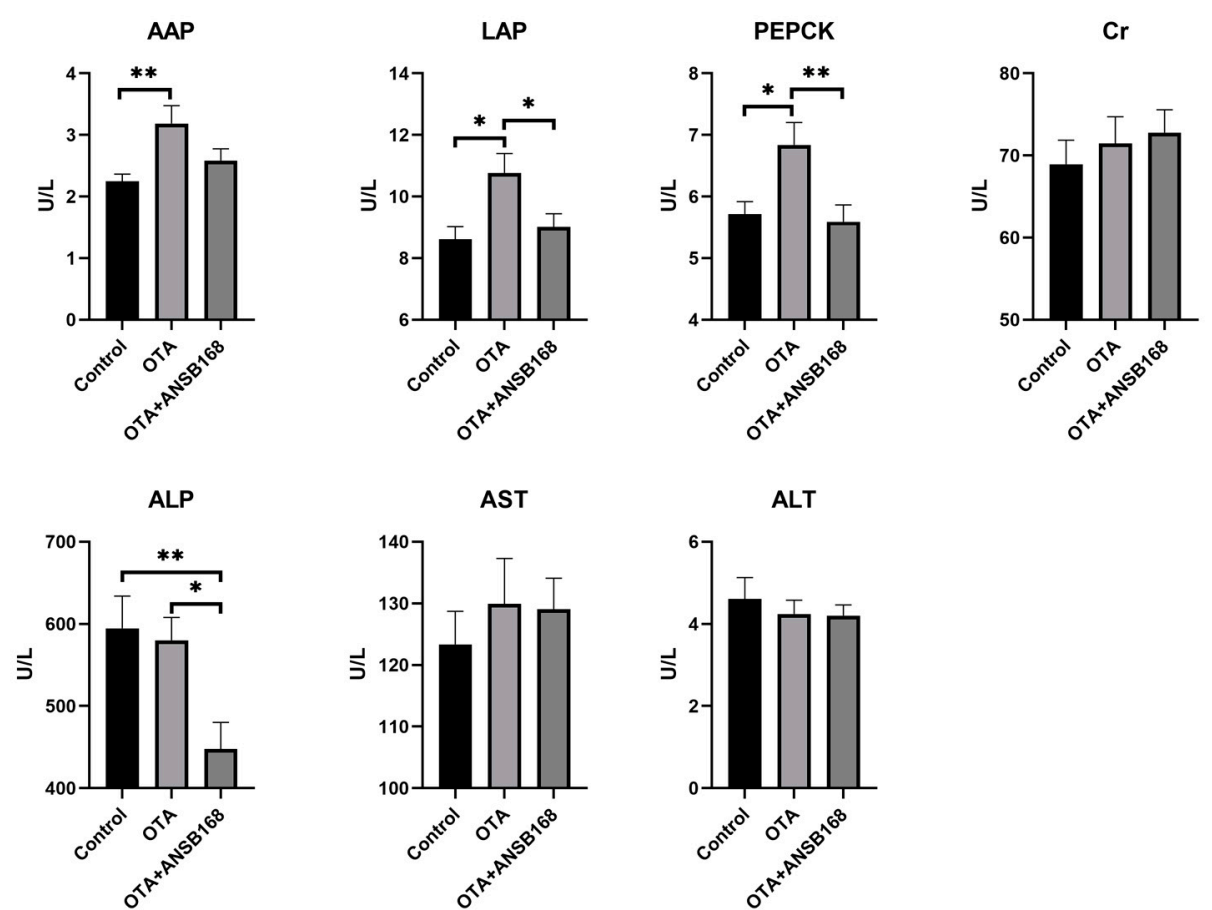

Figure 7. Effects of OTA and ANSB168 on serum biochemical parameters of layers after 28 days ( $n=15$, mean \pm SEM), including AAP, LAP, PEPCK, Cr, ALP, AST, and ALT. Data were analyzed with ANOVA and Tukey's HSD and significant differences were defined as * $p<0.05$, and ${ }^{* *} p<0.01$.

Moreover, the level of LAP $(p<0.05)$, PEPCK $(p<0.01)$, and ALP $(p<0.05)$ in the OTA+ANSB168 group showed a significant decrease compared with that of the OTA-fed group. Levels of AAP also registered a reduction in the OTA+ANSB168 group, though it was not statistically significant. These results indicate that the ANSB168 bioagent could alleviate the damages to the kidney and liver caused by oral OTA, thereby having benefits for layers.

\subsubsection{Oxidative Stress and Antioxidant Status}

The oxidative stress and antioxidant status of OTA-fed and ANSB168-fed layers have been presented in Figure 8. The glutathione reductase (GR) level was significantly higher in the OTA-fed group than in the control group $(p<0.01)$, while the OTA+ANSB168 group showed a decrease compared with the OTA-fed group $(p<0.05)$. Plasma total antioxidant capacity (T-AOC) $(p<0.05)$ and SOD $(p<0.01)$ levels were significantly higher in the OTA+ANSB168 group compared with the other groups. The levels of malonaldehyde (MDA), total glutathione (T-GSH), and GSH-Px differed non-significantly among all groups. These results indicate that $250 \mu \mathrm{g} / \mathrm{kg}$ of OTA in the diet could induce layers' oxidation reaction. However, the layers promoted the regeneration of reduced glutathione by increasing the GR's activity, thereby alleviating its oxidative stress effects. The supplement of ANSB168 could improve the ability of antioxidants by increasing the activity of T-AOC and SOD, therefore leading to ameliorative effects to OTA-fed layers. 


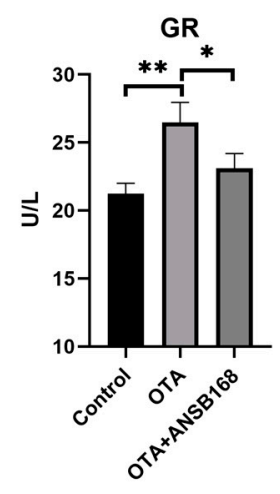

MDA

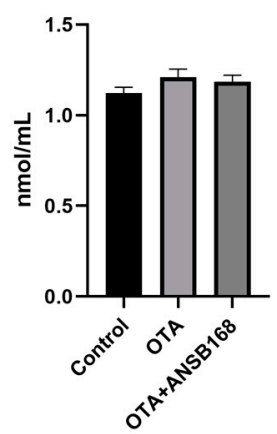

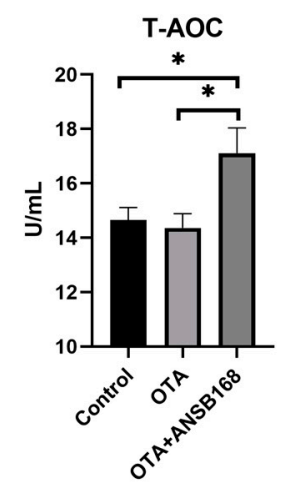

T-GSH

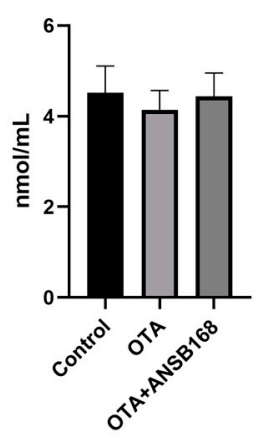

SOD

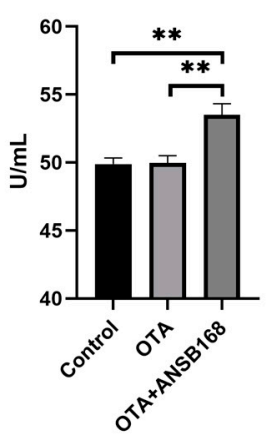

GSH-Px

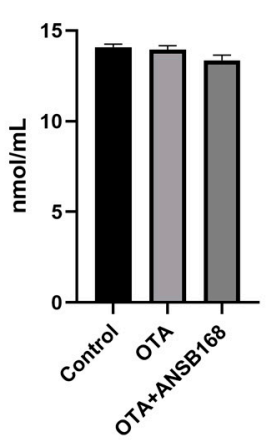

Figure 8. Effects of OTA and ANSB168 on oxidative stress and antioxidant status of layers after 28 days ( $n=15$, mean \pm SEM), including GR, T-AOC, SOD, MDA, T-GSH, and GSH-Px. Data were analyzed with ANOVA and Tukey's HSD and significant differences were defined as * $p<0.05$, and ** $p<0.01$.

\subsubsection{Immune and Inflammatory Response}

Figure 9 exhibited the immune response in layers caused by the OTA and ANSB168 diet. Levels of $\beta 2$-microglobulin ( $\beta 2-\mathrm{MG})(p<0.01)$, immunoglobulin $\mathrm{A}(\operatorname{IgA})(p<0.05)$, immunoglobulin G (IgG) $(p<0.05)$, and lysozyme (LZM) $(p<0.01)$ in the serum after OTA feeding showed a significant increase compared with the control group. In addition, an increasing trend of immunoglobulin $\mathrm{M}(\operatorname{IgM})$ levels $(p=0.065)$ and a decreasing trend of total protein (TP) levels $(p=0.068)$ were observed in OTA-fed layers, compared with the control. Meanwhile, the levels of IgG $(p<0.05)$ and LZM $(p<0.01)$ in the OTA+ANSB168 groups were significantly lower than that of the OTA-fed group. When compared with the OTA-fed group, $\beta 2-\mathrm{MG}$, IgA, and IgM levels in the OTA+ANSB168 groups were lower and the level of TP in the OTA+ANSB168 group was higher. There was no significant difference in albumin (ALB) among all groups.

The inflammatory responses in the layers are shown in Figure 10. In the OTA-fed group, the levels of interleukin-10 (IL-10) and tumor necrosis factors- $\alpha$ (TNF- $\alpha$ ) were significantly higher than those of the control $(p<0.05)$. Apart from that, the concentration of interleukin-2 (IL-2) was higher than that of the control, though it was not statistically significant. Overall, they all increased in the OTA-fed group and decreased in the OTA+ANSB168 group.

These data suggest that the ANSB168 bioagent could alleviate the immune activation effects and inflammatory response caused by oral OTA.

\subsubsection{Residues of OTA and OT $\alpha$ in Eggs}

We did not detect residues of OTA and OT $\alpha$ above the detection limit $(0.1 \mu \mathrm{g} / \mathrm{kg})$ of the HPLC method in the eggs of all groups. 


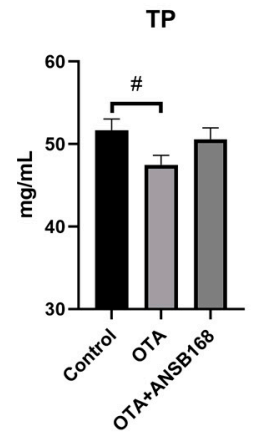

$\lg A$

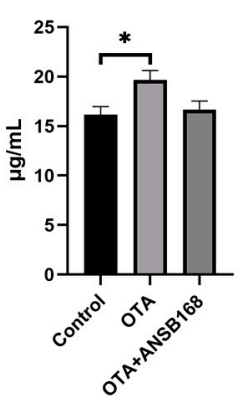

ALB

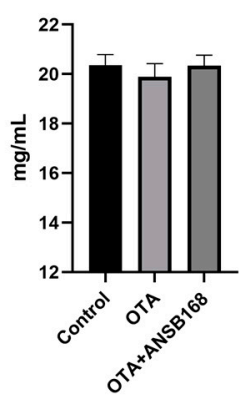

$\lg G$

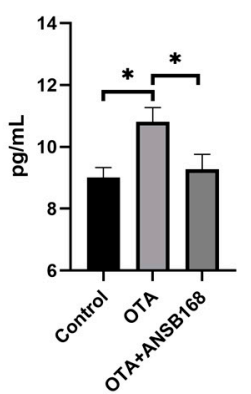

B2-MG
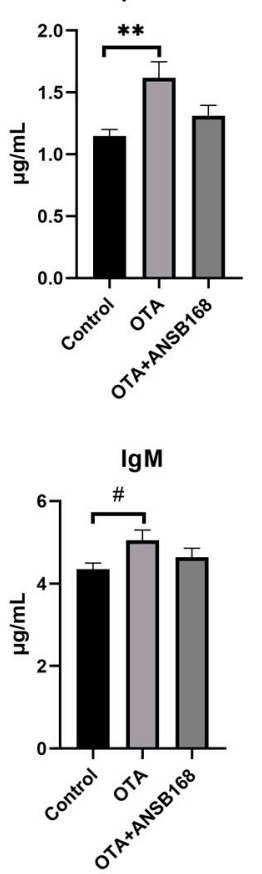

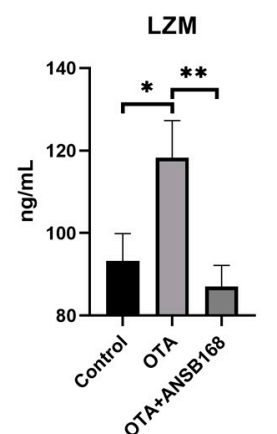

Figure 9. Effects of OTA and ANSB168 on immune response of layers after 28 days $(n=15$, mean $\pm \mathrm{SEM}$ ), including serum immunoglobulin (TP, ALB, ß2-MG, IgA, IgG, IgM) and LZM. Data were analyzed with ANOVA and Tukey's HSD and significant differences were defined as $\# 0.05 \leq p<0.1,{ }^{*} p<0.05$, and ${ }^{* *} p<0.01$.

IL-2

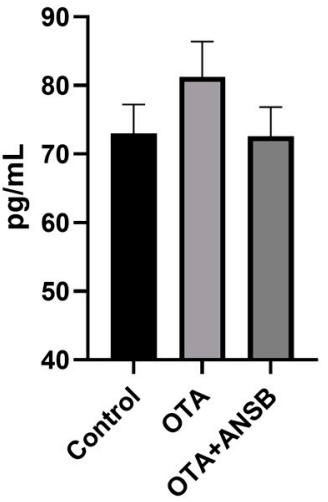

IL-10

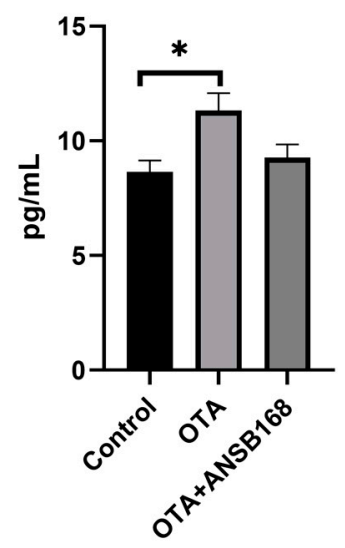

TNF- $\alpha$

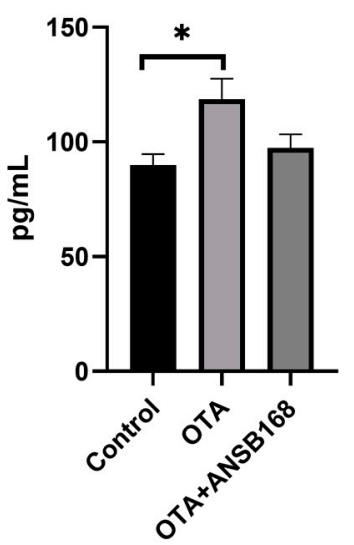

Figure 10. Effects of OTA and ANSB168 on serum cytokines (IL-2, IL-10, and TNF- $\alpha$ ) of layers after 28 days $(n=15$, mean \pm SEM). Data were analyzed with ANOVA and Tukey's HSD and significant differences were defined as $* p<0.05$.

\section{Discussion}

OTA is one of the most significant mycotoxins that widely contaminate agriculture products. OTA contamination in feed seriously affects the health and production performance of animals, which may also harm people through the food chain. Numerous studies have reported that OTA has renal toxicity, liver toxicity, and carcinogenic and teratogenic effects [7-10]. In recent years, biodegradation strategies for OTA detoxification have become a promising method. The ability to degrade, absorb, or bind OTA in different microorganisms species, including Brevibacterium [21], Alcaligenes [22], Aspergillus [23], Saccharomyces [36], Cupriavidus [37], and Lactobacillus [38], has been examined extensively. In previous research, some Brevibacterium spp. strains could degrade $100 \%$ of OTA into the 
nontoxic substance OT $\alpha$ [21]. The strain ANSB168 that we used in this study was initially isolated from the cecum of a donkey and could degrade $47.0 \%$ of OTA to produce OT $\alpha$ within $18 \mathrm{~h}$ in vitro. However, the mentioned reports did not investigate the efficacy of the purified enzyme in OTA degradation. In our experiment, its degradation mechanism and the melioration effects in poultry were further analyzed.

The degradation of OTA into non-toxic or low-toxic metabolites by microorganisms and their intracellular or extracellular enzymes is the current research hotspot of OTA detoxification in feeds and foods. It has been reported that Bacillus amyloliquefaciens ASAG1 [33], Acinetobacter sp. neg1 [39], Bacillus licheniformis CM21 [40], and Alcaligenes faecalis ASAGF 0D-1 [22] can hydrolyze the amide bond in the OTA molecule to generate OT $\alpha$ and Phe. Therefore, it is conjectured that the same type of protease plays the OTA hydrolysis role in these strains. In a previous study, Chang [33] cloned and expressed D-Ala-D-Ala carboxypeptidase from B. amyloliquefaciens ASAG1 and confirmed that D-Ala-D-Ala carboxypeptidase could hydrolyze OTA. In addition, Liuzzi [39] found that adding OTA to the culture medium of Acinetobacter sp. neg1 could up-regulate the expression of D-Ala-D-Ala carboxypeptidase PJ15-1540. Furthermore, PJ15-1540 expressed exogenously in E. coli had OTA degradation activity. The overall results indicate that the D-Ala-D-Ala carboxypeptidase is the potential molecular basis for the bacteria to hydrolyze OTA. In the current study, based on the genomic sequence of D-Ala-D-Ala carboxypeptidases DacA and DacB according to the NCBI database, the strain ANSB168 maintained by the laboratory was used as a template to successfully amplify DacA and DacB by PCR. The method of the E. coli expression system for exogenous expression of DacA and DacB genes was well established. Similarly, Liuzzi [39] successfully cloned the D-Ala-D-Ala carboxypeptidase PJ-1540 derived from Acinetobacter sp. neg1 ITEM 17016 and achieved soluble expression in E. coli. The amino acid sequence of PJ-1540 shared 29\% identity with DacA, while PJ-1540 shared 32\% identity with DacB. D-Ala-D-Ala carboxypeptidase derived from $B$. amyloliquefaciens ASAG1 and expressed in E. coli had the activity of hydrolyzing OTA and inhibiting the growth of OTA-producing A. niger [33]. DacA and DacB, derived from ANSB168 genes, were similar to B. amyloliquefaciens ASAG1 carboxypeptidase (amino acid sequence similarity of $81 \%$ and $35 \%$, respectively).

Although the recombinant proteins DacA and DacB can be successfully expressed in the E. coli expression system, DacA and DacB were partly in the form of inclusion bodies. There are two possible reasons for the formation of inclusion bodies. Firstly, the recombinant proteins DacA and DacB may be expressed too quickly to fold correctly, resulting in the generation of the hydrophobic domain. Secondly, the existence of E. coli may have side effects on the proteins DacA and DacB. We only used cell-free soluble recombinant proteins in the supernatant for purification because the inclusion bodies need to be denatured and then renatured in a purification process, which is inefficient and may reduce enzyme activity. The expression levels of DacA and DacB were 10.26 and 9.24 mg/L, respectively, which were higher than the $4 \mathrm{mg} / \mathrm{L}$ expressions of bovine pancreatic CPA zymogen in E. coli [41]. Since the carboxyl end of the recombinant protein carries a His-tag composed of six histidines, the mouse anti-His monoclonal antibody could be employed to identify whether the purified recombinant protein is the target protein. Western blot analysis found that the purified DacA and DacB can be specifically recognized by the mouse anti-His monoclonal antibody, thus further confirming the successful expression and purification of DacA and DacB in this experiment.

The results showed that D-Ala-D-Ala carboxypeptidase DacA and DacB derived from ANSB168 had OTA degradation activity. Under the optimum conditions, DacA and DacB can hydrolyze $45 \%$ and $42 \%$ of OTA, respectively, after $72 \mathrm{~h}$ of incubation. This degradation ratio was higher than the 33\% degradation of OTA by the Neg1 D-Ala-D-Ala carboxypeptidase PJ15-1540 crude enzyme derived from Acinetobacter sp. neg1 [39]. Likewise, the D-Ala-D-Ala carboxypeptidase originated from B. amyloliquefaciens ASAG1 could degrade $41 \%$ of OTA at $28{ }^{\circ} \mathrm{C}$ after $12 \mathrm{~h}$ of incubation [33], while the carboxypeptidase cp4 derived from Lysobacter sp. CW239 could degrade 36.8\% of OTA after $24 \mathrm{~h}$ of incubation [35]. 
The Michaelis constant $\left(K_{\mathrm{m}}\right)$ can reflect the affinity of an enzyme for its substrate, while $V_{\text {max }}$ represents the rate of an enzyme-catalyzed reaction when the substrate concentration reaches saturation [42]. In the present study, the $K_{m}$ value of DacA and DacB hydrolyzing OTA were $2.74 \mu \mathrm{g} / \mathrm{mL}$ and $1.14 \mu \mathrm{g} / \mathrm{mL}$, respectively. The calculated values of the $V_{\max }$ of DacA and DacB hydrolyzing OTA were $73.53 \mathrm{ng} / \mathrm{h} / \mathrm{mg}$ and $42.74 \mathrm{ng} / \mathrm{h} / \mathrm{mg}$, respectively. In general, the affinity of DacB with OTA was greater than that of DacA, while DacA had a higher reaction velocity than DacB.

Determining the structure and toxicity of the degradation products is the key to evaluating whether the mycotoxin-degrading bacteria or enzymes can be used in actual production. OT $\alpha$ is one of the most ideal degradation products that has been widely reported $[21,23,43]$. Compared with the control group, the DacA and DacB treatment groups had a degradation product peak with a retention time of about $6.7 \mathrm{~min}$. The retention time of the product is consistent with the retention time of OT $\alpha$ standard. Therefore, it was confirmed that DacA and DacB could hydrolyze the amide bond of OTA to generate low-toxic OT $\alpha$. Likewise, a series of carboxypeptidases derived from different strains, including B. amyloliquefaciens ASAG1 [33], Lysobacter sp. CW239 [35], and Acinetobacter sp. neg1 [39] could also degrade OTA into OT $\alpha$. On the whole, the results elucidated the degradation mechanism of the newly isolated strain ANSB168. It is confirmed that the intracellular enzyme D-Ala-D-Ala carboxypeptidase DacA and DacB can hydrolyze OTA on the amide bond into less toxic OT $\alpha$, which makes it necessary to evaluate the actual use of ANSB168 in an in vivo animal test to develop a novel OTA detoxification biological product in the future.

Stoev [44] reported that the egg production number and egg weight were significantly reduced by $10.77 \%$ and $3.49 \%$, respectively, in OTA-fed laying hens with $1 \mathrm{mg} / \mathrm{kg}$ feed. Likewise, an OTA-contaminated diet could significantly reduce young chickens' body weight, average daily gain (g/d), and daily feed intake, even at low levels $(100 \mu \mathrm{g} / \mathrm{kg}$ feed) [45]. Similar results were obtained with a $100 \mu \mathrm{g} / \mathrm{kg}$ OTA-contaminated diet in breeder hens, where their body weight, feed intake, and egg production (g) significantly decreased by $1.85 \%, 0.81 \%$, and $18.41 \%$, respectively [46]. In the animal trial, we intended to investigate the amelioration effects of the freeze-dried bacterial culture powder of ANSB168 $\left(3 \times 10^{7} \mathrm{CFU} / \mathrm{g}, 2 \mathrm{~kg} / \mathrm{T}\right.$ feed $)$ in OTA-exposure poultry. The dose of OTA fed to the laying hens was $250 \mu \mathrm{g} / \mathrm{kg}$ of feed, which is higher than the recommended upper limit in commercial poultry feed in Europe $(100 \mu \mathrm{g} / \mathrm{kg}$ feed, 2006/576/EC). We tested the production performances and serum parameters in laying hens after administering OTA and/or freeze-dried ANSB168 for 28 days. The results showed that OTA had negative effects on laying hens' production performances, including egg production ratio, daily egg production, feed/egg ratio, and average egg weight, though these were not statistically significant due to the variable differences within groups. Plus, the average daily feed intake was significantly lower than that of the control. These results are consistent with those of other studies. In the present study, the supplementation of ANSB168 represented ameliorative effects on layers' production performances.

Parameters such as the serum concentrations of several proteins and metabolites and the activity of certain enzymes can be used as sensitive indicators of ochratoxin exposure [47]. Biochemical signs of ochratoxin toxicity reported in the literature in poultry include decreases in cholesterol, TP, ALB, globulin, potassium, and triglyceride levels, and increases in uric acid, creatine levels, activities of serum ALP, and GGT [48]. In the current study, significant increases were observed in serum AAP, LAP, PEPCK, GR, $\beta 2-\mathrm{MG}, \operatorname{IgA}$, IgG, LZM, IL-10, and TNF- $\alpha$ concentration in birds exposed to OTA in the diet, indicating that OTA induced kidney damage, oxidative stress, immune response, and inflammation. However, the bioproduct of ANSB168 can significantly reduce the concentration of LAP, PEPCK, GR, IgG, and LZM and increase the concentration of T-AOC and SOD, suggesting a degradation effect of OTA-contaminated feed inside layers' bodies.

It has been reported that OTA was not detectable in the eggs when laying hens were exposed to a $10 \mu \mathrm{g} / \mathrm{kg}$ and $200 \mu \mathrm{g} / \mathrm{kg}$ OTA diet [49]. After administration of $2 \mathrm{mg} / \mathrm{kg}$ 
of OTA in feed, the residues of OTA in the eggs were still not detectable $[48,50]$. We did not detect residues of OTA and OT $\alpha$ above the detection limit $(0.1 \mu \mathrm{g} / \mathrm{kg})$ with the HPLC method in the eggs of all groups, which is in line with other studies. However, a conflicting report detected 1 to $8 \mu \mathrm{g} / \mathrm{kg}$ of OTA residues in eggs after administrating a $500 \mu \mathrm{g} / \mathrm{kg}$ and $5 \mathrm{mg} / \mathrm{kg}$ OTA diet in laying hens [51]. A great deviation between each analysis was obtained in the conflicting report, suggesting that the OTA passage rate into eggs between different individuals varied significantly.

Except for the crop and gizzard, the $\mathrm{pH}$ of poultrys' digestive tract is above 5.0 [52]. In the present study, the optimal $\mathrm{pH}$ for DacA and DacB degrading OTA was between 6.5 and 7.5, which happened to be the range of $\mathrm{pH}$ of the small intestine. The passage time for $50 \%$ relative cumulation of dry excreta through the poultrys' total digestive tract was about $32 \mathrm{~h}$ [53]. The retention time of dry matter in the crop and gizzard only took up $27-33 \%$ of the whole gastrointestinal tract emptying time. Considering that digesta could be held in the small intestine for the longest time [54], we deduced that the degradation of OTA by ANSB168 was mainly performed in the small intestine. The degradation activity of DacA and DacB was inhibited at pH below 5.0 and increased with the increase of $\mathrm{pH}$, which demonstrated that the degradation activity of ANSB168 was inhibited in the crop and gizzard and could be reactivated in the intestinal tract.

\section{Materials and Methods}

\subsection{Chemicals and Strains}

OTA standard and OT $\alpha$ standard were purchased from Sigma (St. Louis, MO, USA) and were prepared in HPLC grade methanol. The strain ANSB168 was initially isolated from donkey cecum and maintained with $20 \%$ glycerin at $-20^{\circ} \mathrm{C}$ in our lab. E. coli DH5 $\alpha$ and E. coli Rosetta (DE3) were purchased from Sangon Biotech (Shanghai, China). The pET-31b vector was obtained from Novagen (Madison, WI, USA). The strain Aspergillus ochraceus 3.4412 was purchased from CGMCC (Beijing, China).

\subsection{Enrichment and Isolation of OTA-Degrading Bacteria from Donkey Cecum}

Ten samples were collected from the cecum of different donkeys. About $1 \mathrm{~g}$ of each sample was suspended in $9 \mathrm{~mL}$ of sterile distilled water and kept at room temperature under continuous shaking at $200 \mathrm{rpm}$ for $6 \mathrm{~h}$. Then, the supernatant was serially diluted in sterile distilled water, and a $150 \mu \mathrm{L}$ aliquot of each dilution was spread on plates with LB medium and incubated at $37^{\circ} \mathrm{C}$ until visible colonies appeared. The single colonies were isolated and subsequently streaked on fresh plates to obtain pure cultures. Then, the cultures were tested for OTA degradation capacity. For bacterial OTA degradation, a final concentration of $2 \mu \mathrm{g} / \mathrm{mL}$ OTA was mixed with a $500 \mu \mathrm{L}$ LB broth containing $1 \times 10^{8} \mathrm{CFU} / \mathrm{mL}$ of ANSB168 and incubated for $18 \mathrm{~h}$ at $37^{\circ} \mathrm{C}$. After incubation, OTA was analyzed by HPLC. After analysis, the strain ANSB168 showed the highest OTA degradation ability, so it was chosen for further studies.

\subsection{Cloning of D-alanyl-D-alanine Carboxypeptidase DacA and DacB}

Genomic DNA of the strain ANSB168 was isolated using a Bacterial Genomic DNA Extraction Kit (Transgen, China). Then, it was used as a template for cloning and the D-alanyl-D-alanine carboxypeptidase DacA and DacB genes were amplified by PCR. A primer pair of 5'-GTAGATTCATATGGCCAGCGATCC-3' (forward primer) and 5'CATCTCGAGAAACCAGCCGGTTA-3' (reverse primer) was used for DacA, while another primer pair of 5'-GCTTATTCATATGGCTATAGATGTC-3' (forward primer) and 5'-CATCTCGAGTATTGACCATTTTG-3' (reverse primer) was employed for DacB. With the same restriction enzyme sites of NdeI and XhoI (underlined), the PCR amplification of these two carboxypeptidases was carried out separately. The conditions of PCR were 3 min initial denaturing at $9{ }^{\circ} \mathrm{C}$, followed by 35 cycles of $30 \mathrm{~s}$ denaturing at $95{ }^{\circ} \mathrm{C}, 30 \mathrm{~s}$ annealing, and $90 \mathrm{~s}$ extension at $72{ }^{\circ} \mathrm{C}$, and then a final extension at $72{ }^{\circ} \mathrm{C}$ lasting for $15 \mathrm{~min}$. A TIANgel Midi Purification Kit (Tiangen, Beijing, China) was used to recycle the PCR 
product. By using an Omega D6943-01 Plasmid Mini Kit I (Tiangen, Beijing, China), the pET-31b vector was digested by NdeI and XhoI. The PCR products were also digested by the same restriction sites, and then they were cloned into digested $\mathrm{pET}-31 \mathrm{~b}$ vectors (T4 DNA Ligase, New England Biolabs, MA, USA). After being authenticated in the E. coli $\mathrm{DH} 5 \alpha$ strain, the selected colonies were transformed into an E. coli Rosetta strain (DE3).

\subsection{Heterologous Expression and Protein Purification}

The manipulated cells with $\mathrm{pET}-31 \mathrm{~b}-\mathrm{DacA} / \mathrm{pET}-31 \mathrm{~b}-\mathrm{DacB}$ vectors were cultured in LB broth (HuanKai, Guangdong, China) at $3^{\circ} \mathrm{C}$ with an agitation of $180 \mathrm{rpm}$, and then IPTG was added to the broth with a cell at $\mathrm{OD}_{600} 0.6$ to induce for $4 \mathrm{~h}$. The fermentation broth was centrifuged at $12,000 \mathrm{rpm}$ for $30 \mathrm{~min}$ and redissolved in binding buffer, followed by ultrasonication and $10 \mathrm{~min}$ of centrifugation, and then the supernate and sediment were collected separately. The overexpression of the target protein was measured by using (SDS-PAGE. Because the recombinant protein has a His-tag, a nickel-nitrilotriacetic acidagarose resin (Ni-NTA, Qiagen, CA, USA) was applied based on affinity chromatography for purification. After loading the sample, the column was purified by a different portion of binding buffer ( $50 \mathrm{mM}$ sodium phosphate, $500 \mathrm{mM} \mathrm{NaCl}, 50 \mathrm{mM}$ imidazole, $\mathrm{pH} 7.4$ ) and elution buffer ( $50 \mathrm{mM}$ sodium phosphate, $500 \mathrm{mM} \mathrm{NaCl}, 300 \mathrm{mM}$ imidazole, $\mathrm{pH}$ 7.4). The purified protein was analyzed by SDS-PAGE as well as Western blotting and concentrated by ultrafiltration.

\subsection{SDS-PAGE and Western Blot Analysis}

SDS-PAGE and Western blot analysis were conducted by previously reported methods [55]. The first antibody was a mouse anti-His antibody, and the second antibody was goat anti-mouse IgG $(\mathrm{H}+\mathrm{L})$-horseradish peroxidase (HRP, 1:2000, Abcam, Cambridge, UK).

\subsection{OTA Degradation Activity and Relevant Enzyme Characteristics}

OTA working solution was prepared using pure methanol and the final concentration was $2 \mu \mathrm{g} / \mathrm{mL}$. The recombinant proteins with the final concentration of $400 \mu \mathrm{g} / \mathrm{mL}$ were added into different degradation systems. The reaction system had a $500 \mu \mathrm{L}$ volume consisting of $10 \mu \mathrm{L}$ of OTA standard $(100 \mu \mathrm{g} / \mathrm{mL}$ in methanol) and $490 \mu \mathrm{L}$ of reagents. The reaction system without recombinant DacA and $\mathrm{DacB}$ was regarded as a negative control. To determine the optimal $\mathrm{pH}$, varying $\mathrm{pH}$ values $(5.5,6.0,6.5,7.0,7.5,8.0,8.5)$ were selected in $0.1 \mathrm{M}$ buffer (citric acid-sodium citrate buffer, $\mathrm{pH}$ : 5.5-6.5; disodium hydrogen phosphate-sodium dihydrogen phosphate buffer, $\mathrm{pH}$ : 6.5-8.5). At the optimal $\mathrm{pH}$ DacA or DacB, a $500 \mu \mathrm{L}$ volume reaction system was applied to assess optimal temperature $\left(27^{\circ} \mathrm{C}, 32{ }^{\circ} \mathrm{C}, 37^{\circ} \mathrm{C}, 42{ }^{\circ} \mathrm{C}, 47^{\circ} \mathrm{C}\right)$. The degradation solution had a $500 \mu \mathrm{L}$ volume and was incubated in total darkness for $72 \mathrm{~h}$ at $37^{\circ} \mathrm{C}$; then, $500 \mu \mathrm{L}$ of HPLC grade methanol was used to terminate the reaction. The OTA degradation dynamic was explored at optimal $\mathrm{pH}$ and temperature and incubating for 5 days. The OTA degradation ability and degraded product were analyzed using HPLC.

\subsection{Dietary Treatments of Animal Trial}

The trial was conducted with a basal diet that was used as a negative control and also to produce the OTA contaminated dietary treatments. An A. ochraceus (CGMCC 3.4412) strain was used to produce OTA by artificial infection of sterile maize for $21 \mathrm{~d}$ at $25-28{ }^{\circ} \mathrm{C}$, and then the maize was dried and smashed. The concentration of OTA in maize powder was measured by HPLC, which was later added into the basal diet at $18 \%$ to meet the predicted concentration and verified by HPLC (predicted: $250 \mu \mathrm{g} / \mathrm{kg}$, measured: $247.8 \mu \mathrm{g} / \mathrm{kg}$ ). The broth of strain ANSB168 was transformed into a freeze-dried powder. The number of bacteria in the powder was later determined as $3 \times 10^{7} \mathrm{CFU} / \mathrm{g}$. Then, it was added to the contaminated basal diet up to an overdose of $2 \mathrm{~kg} / \mathrm{T}$ feed to ensure the effectiveness of degradation. 


\subsection{Animal Trial in Layers}

All procedures were reviewed and approved by the Laboratory Animal Welfare and Animal Experimental Ethical Committee of China Agricultural University (No. AW 13301202-1-7). The trial strictly complied with the standard operating procedures for experimental animals of the Ministry of Science and Technology (Beijing, China), and every effort was made to minimize suffering.

A total of 45 Jingfen No. 1 layers (26 weeks of age) were randomly allocated to three feeding treatments and divided between 15 pens. The nutritive values and feeding procedures referred to the NY/T 33-2004 (China) and recommendations for Jingfen No. 1 layers (Huadu yukou, Beijing, China). For the first 7 days, all birds were fed a basal diet. From day 8 onwards, the birds on the control diet continued a basal diet while the other treatment groups received a targeted $250 \mu \mathrm{g} / \mathrm{kg}$ OTA-contaminated diet. An amount of $2 \mathrm{~kg} / \mathrm{T}$ freeze-dried bacterial powder (ANSB168) was added to one of the OTA-exposure groups. The production performance of laying hens was continuously calculated over a period of 1-28 days.

\subsection{Analysis of Serum Parameters}

The contents of AST, ALT, ALP, PEPCK, Cr, TP, ALB, LZM, T-AOC, SOD, and GR in serum were measured using diagnostic kits (Nanjing Jiancheng Bioengineering Institute, Nanjing, China) according to the manufacturer's instructions. The activity of LAP and AAP, as well as the levels of MDA, T-GSH, GSH-Px, globulin ( $\beta 2-\mathrm{MG}$, IgA, IgG, IgM), and cytokines (TNF- $\alpha$, IL-2 and IL-10) in the serum were measured with the ELISA kit (Nanjing Jiancheng Bioengineering Institute, Nanjing, China). All procedures were performed according to the manufacturer's instructions.

\subsection{Extration and Clean-Up of OTA in Feed and Eggs}

Levels of OTA in feed were determined before the animal trial. Residues of OTA and OT $\alpha$ in eggs were determined weekly during the animal trial, i. e., days 14, 21, and 28. OTA extraction from feed and eggs was conducted according to the methods of previous studies $[49,56]$. Then, extracted samples were passed through immunoaffinity clean-up columns (OchraTestWB; VICAM, Watertown, MA, USA) at a rate of 1-2 drops $\mathrm{s}^{-1}$ under gentle pressure provided by a vacuum clean-up assembly. The column was washed with $10 \mathrm{~mL}$ of water-methanol $(90: 10, v / v)$ and then dried under nitrogen gas $\left(\mathrm{N}_{2}\right)$ for $5 \mathrm{~min}$. Finally, OTA was eluted from the column by passing $2.0 \mathrm{~mL}$ of pure methanol [57].

\subsection{High-Performance Liquid Chromatography}

The contents of OTA and OT $\alpha$ were determined using an HPLC system (Shimadzu LC-10 AT, Shimadzu, Tokyo, Japan) equipped with an Agilent ${ }^{\circledR}$ Eclipse Plus C18 column $(5 \mu \mathrm{m}, 4.6 \times 150 \mathrm{~nm})$ and a fluorescence detector (Shimadzu RF-20A, Shimadzu, Tokyo, Japan). Samples were filtered using a $0.22 \mu \mathrm{m}$ filter and $20 \mu \mathrm{L}$ of volume was loaded to the HPLC system. OTA and OT $\alpha$ detection used $333 \mathrm{~nm}$ and $477 \mathrm{~nm}$ as the wavelengths of excitation and emission, respectively. The mobile phase consisted of acetonitrile-waterglacial acetic acid (99:99:2, v/v/v), and the flow rate was $1 \mathrm{~mL} \mathrm{~min}^{-1}$ [57]. The limits of detection in feed and egg samples (defined as signal/noise ratio $=3$ ) were estimated to be $0.1 \mu \mathrm{g} / \mathrm{kg}$ for both OTA and OT $\alpha$.

\subsection{Statistical Analysis}

Statistically significant differences between mean values of the parameters tested in the animal trial were analyzed with ANOVA using Tukey's honestly significant difference (HSD) post-hoc test in the SPSS statistical software package (version 22, Armonk, NY, USA). The differences were considered statistically significant if the $p$ values $<0.05$, although $p$ values $<0.10$ have also been used to demonstrate a trend because of the small number of subjects. The GraphPad Prism software for Windows was used to generate graphs (version 900, San Diego, CA, USA). 


\title{
5. Conclusions
}

In summary, we elucidated the mechanism of an isolated OTA-degrading strain Bacillus subtilis ANSB168 by cloning and expressing the D-Ala-D-Ala carboxypeptidase DacA and DacB from ANSB168, which broke the amide bond of OTA to generate low-toxic metabolites OT $\alpha$. In the animal trial, $250 \mu \mathrm{g} / \mathrm{kg}$ of OTA in the diet caused significant damage to the kidney and liver, induced oxidative stress, caused obvious inflammation, and activated the humoral immune system in the layers, which led to a decline in animal fitness. OTA degradation agent ANSB168 bioproducts were able to alleviate OTA-induced kidney and liver damage; relieve OTA-induced oxidative stress, immune response, and inflammation; and ultimately improve the health of animals. The detoxification mechanism of the strain ANSB168 involves mainly reducing the content of OTA by degrading mycotoxin in the intestine, thus alleviating its negative effects. The optimization of the ANSB168 bioproducts needs to be further developed in the future.

Author Contributions: Conceptualization, H.Q., X.H., and Q.M.; methodology, H.Q., X.H., S.H., and Q.M.; formal analysis and data curation, H.Q., X.H., and S.H.; writing—original draft preparation, H.Q. and X.H.; writing—review and editing, H.Q., Q.M., L.Z., J.Z., and C.J.; validation and supervision, L.Z., and S.H.; project administration and funding acquisition, Q.M. All authors have read and agreed to the published version of the manuscript.

Funding: This research was funded by Beijing Municipal Science Foundation (No. 6172017), Anhui Province Key Laboratory of Livestock and Poultry Product Safety Engineering (No. XM2004), and China Agricultural Research System program (CARS-40-K08).

Institutional Review Board Statement: The study was conducted according to the guidelines for experimental animals of the Ministry of Science and Technology (Beijing, China), and approved by the Laboratory Animal Welfare and Animal Experimental Ethical Committee of China Agricultural University (No. AW 13301202-1-7, 25 November 2019).

Data Availability Statement: Publicly available datasets were analyzed in this study. This data can be found here: https:/ /www.ncbi.nlm.nih.gov/nuccore/OK663194.1/, accessed on 4 November 2021.

Acknowledgments: We thank all the researchers at our laboratory for their help with samples collection. Hanrui Qing and Xueting Huo contributed equally to this work.

Conflicts of Interest: The authors declare no conflict of interest.

\begin{abstract}
Abbreviations
OTA: ochratoxin A; OT $\alpha$ : ochratoxin $\alpha$; Phe: L- $\beta$-phenylalanine; CPA: carboxypeptidase A; CPB: carboxypeptidase B; HPLC: high-performance liquid chromatography; NCBI: National Center for Biotechnology Information; IPTG: isopropyl-b-D-thiogalactoside; SDSPAGE: sodium dodecyl sulfate-polyacrylamide gel electrophoresis; LAP: leucine aminopeptidase; AAP: alanine aminopeptidase; PEPCK: phosphoenolpyruvate carboxykinase; $\mathrm{Cr}$ : creatinine; AST: aspartate aminotransferase; ALT: alanine aminotransferase; ALP: alkaline phosphatase; MDA: malonaldehyde; T-GSH: total glutathione; GSH-Px, glutathione peroxidase; T-AOC: total antioxidant capacity; SOD: superoxide dismutase; TAS: total antioxidant status; GR: glutathione reductase; TP: total protein; ALB: albumin; $\beta 2-\mathrm{MG}$ : $\beta 2-$ microglobulin; IgA, IgG, IgM: immunoglobulin A, G, M; LZM: lysozyme; IL-2: cytokines like interleukin-2; IL-10: interleukin-10; TNF- $\alpha$ : tumor necrosis factors- $\alpha$.
\end{abstract}

\section{References}

1. Van Der Merwe, K.J.; Steyn, P.S.; Fourie, L.; Scott, D.B.; Theron, J.J. Ochratoxin A, a Toxic Metabolite produced by Aspergillus ochraceus Wilh. Nature 1965, 205, 1112-1113. [CrossRef] [PubMed]

2. Abarca, M.L.; Bragulat, M.R.; Castella, G.; Cabañes, F.J. Ochratoxin A production by strains of Aspergillus niger var. niger. Appl. Environ. Microbiol. 1994, 60, 2650-2652. [CrossRef]

3. Téren, J.; Varga, J.; Hamari, Z.; Rinyu, E.; Kevei, F. Immunochemical detection of ochratoxin A in black Aspergillus strains. Mycopathologia 1996, 134, 171-176. [CrossRef] 
4. Visagie, C.M.; Varga, J.; Houbraken, J.; Meijer, M.; Kocsubé, S.; Yilmaz, N.; Fotedar, R.; Seifert, K.A.; Frisvad, J.C.; Samson, R.A. Ochratoxin production and taxonomy of the yellow aspergilli (Aspergillus section Circumdati). Stud. Mycol. 2014, 78, 1-61. [CrossRef]

5. Larsen, T.O.; Svendsen, A.; Smedsgaard, J. Biochemical characterization of ochratoxin a-producing strains of the genus penicillium. Appl. Environ. Microbiol. 2001, 67, 3630-3635. [CrossRef] [PubMed]

6. Cabañes, F.J.; Bragulat, M.R.; Castellá, G. Ochratoxin A Producing Species in the Genus Penicillium. Toxins 2010, 2, 1111-1120. [CrossRef]

7. Akpinar, H.A.; Kahraman, H.; Yaman, I. Ochratoxin a sequentially activates autophagy and the ubiquitin-proteasome System. Toxins 2019, 11, 615. [CrossRef] [PubMed]

8. Imaoka, T.; Yang, J.; Wang, L.; McDonald, M.G.; Afsharinejad, Z.; Bammler, T.K.; Van Ness, K.; Yeung, C.K.; Rettie, A.E.; Himmelfarb, J.; et al. Microphysiological system modeling of ochratoxin A-associated nephrotoxicity. Toxicology 2020, 444, 152582. [CrossRef]

9. Li, P.; Li, K.; Zou, C.; Tong, C.; Sun, L.; Cao, Z.; Yang, S.; Lyu, Q. Selenium Yeast Alleviates Ochratoxin A-Induced Hepatotoxicity via Modulation of the PI3K/AKT and Nrf2/Keap1 Signaling Pathways in Chickens. Toxins 2020, 12, 143. [CrossRef]

10. Le, G.; Yuan, X.; Hou, L.; Ge, L.; Liu, S.; Muhmood, A.; Liu, K.; Lin, Z.; Liu, D.; Gan, F.; et al. Ochratoxin A induces glomerular injury through activating the ERK/NF-KB signaling pathway. Food Chem. Toxicol. 2020, 143, 111516. [CrossRef]

11. Damiano, S.; Andretta, E.; Longobardi, C.; Prisco, F.; Paciello, O.; Squillacioti, C.; Mirabella, N.; Florio, S.; Ciarcia, R. Effects of Curcumin on the Renal Toxicity Induced by Ochratoxin A in Rats. Antioxidants 2020, 9, 332. [CrossRef] [PubMed]

12. Marin, D.E.; Pistol, G.; Gras, M.A.; Palade, M.L.; Taranu, I. Comparative effect of ochratoxin A on inflammation and oxidative stress parameters in gut and kidney of piglets. Regul. Toxicol. Pharmacol. 2017, 89, 224-231. [CrossRef] [PubMed]

13. Marin, D.E.; Motiu, M.; Pistol, G.C.; Gras, M.A.; Israel-Roming, F.; Calin, L.; Stancu, M.; Taranu, I. Diet contaminated with ochratoxin A at the highest level allowed by EU recommendation disturbs liver metabolism in weaned piglets. World Mycotoxin J. 2016, 9, 587-596. [CrossRef]

14. Hameed, M.R.; Khan, M.Z.; Saleemi, M.K.; Khan, A.; Akhtar, M.; Hassan, Z.-U.; Hussain, Z. Study of ochratoxin A (OTA)-induced oxidative stress markers in broiler chicks. Toxin Rev. 2017, 36, 270-274. [CrossRef]

15. Khatoon, A.; Khan, M.Z.; Abidin, Z.U.; Bhatti, S.A. Effects of feeding bentonite clay upon ochratoxin A-induced immunosuppression in broiler chicks. Food Addit. Contam. Part A 2018, 35, 538-545. [CrossRef] [PubMed]

16. Raters, M.; Matissek, R. Thermal stability of aflatoxin B1 and ochratoxin A. Mycotoxin Res. 2008, 24, 130-134. [CrossRef]

17. Varga, J.; Rigo, K.; Teren, J.; Mesterhazy, A. Recent advances in ochratoxin research-I. Production, detection and occurrence of ochratoxins. Cereal Res. Commun. 2001, 29, 85-92. [CrossRef]

18. Ameer Sumbal, G.; Hussain Shar, Z.; Hussain Sherazi, S.T.; Sirajuddin; Nizamani, S.M.; Mahesar, S.A. Decontamination of poultry feed from ochratoxin A by UV and sunlight radiations. J. Sci. Food Agric. 2016, 96, 2668-2673. [CrossRef]

19. Rajendran, R.M.; Umesh, B.; Chirakkal, H. Assessment of H- $\beta$ zeolite as an ochratoxin binder for poultry. Poult. Sci. 2020, 99, 76-88. [CrossRef]

20. Zeidan, R.; Ul-Hassan, Z.; Al-Thani, R.; Migheli, Q.; Jaoua, S. In-Vitro Application of a Qatari Burkholderia cepacia strain (QBC03) in the Biocontrol of Mycotoxigenic Fungi and in the Reduction of Ochratoxin A biosynthesis by Aspergillus carbonarius. Toxins 2019, 11, 700. [CrossRef]

21. Rodriguez, H.; Reveron, I.; Doria, F.; Costantini, A.; Rivas, B.D.L.; Muňoz, R.; Garcia-Moruno, E. Degradation of Ochratoxin A by Brevibacterium Species. J. Agric. Food Chem. 2011, 59, 10755-10760. [CrossRef] [PubMed]

22. Zhang, H.; Wang, Y.; Zhao, C.; Wang, J.; Zhang, X. Biodegradation of ochratoxin A by Alcaligenes faecalisisolated from soil. J. Appl. Microbiol. 2017, 123, 661-668. [CrossRef] [PubMed]

23. Xiong, K.; Zhi, H.-W.; Liu, J.-Y.; Wang, X.-Y.; Zhao, Z.-Y.; Pei, P.-G.; Deng, L.; Xiong, S.-Y. Detoxification of Ochratoxin A by a novel Aspergillus oryzae strain and optimization of its biodegradation. Rev. Argentina Microbiol. 2021, 53, 48-58. [CrossRef] [PubMed]

24. Varga, J.; Rigó, K.; Téren, J. Degradation of ochratoxin A by Aspergillus species. Int. J. Food Microbiol. 2000, 59, 1-7. [CrossRef]

25. Jiang, C.; Shi, J.; Liu, Y.; Zhu, C. Inhibition of Aspergillus carbonarius and fungal contamination in table grapes using Bacillus subtilis. Food Control 2014, 35, 41-48. [CrossRef]

26. Ammar, H.; El-Desouky, T. Green synthesis of nanosilver particles by Aspergillus terreus HA1N and Penicillium expansum HA2N and its antifungal activity against mycotoxigenic fungi. J. Appl. Microbiol. 2016, 121, 89-100. [CrossRef]

27. Hadidane, R.; Bacha, H.; Creppy, E.; Hammami, M.; Ellouze, F.; Dirheimer, G. Isolation and structure determination of natural analogues of the mycotoxin ochratoxin A produced by Aspergillus ochraceus. Toxicology 1992, 76, 233-243. [CrossRef]

28. Marín, S.; Ramos, A.J.; Cano-Sancho, G.; Sanchis, V. Mycotoxins: Occurrence, toxicology, and exposure assessment. Food Chem. Toxicol. 2013, 60, 218-237. [CrossRef]

29. Parker, R.W.; Phillips, T.D.; Kubena, L.F.; Russell, L.H.; Heidelbaugh, N.D. Inhibition of pancreatic carboxypeptidase A: A possible mechanism of interaction between penicillic acid and ochratoxin A. J. Environ. Sci. Health Part B 1982, 17, 77-91. [CrossRef]

30. Xiong, L.; Peng, M.; Zhao, M.; Liang, Z. Truncated expression of a carboxypeptidase a from bovine improves its enzymatic properties and detoxification efficiency of ochratoxin A. Toxins 2020, 12, 680. [CrossRef]

31. Ma, L.; Qiu, X.; Li, Y.; Tang, S.; Shen, W.; Xing, C.; Kong, D.; Sheng, J. Carboxypeptidase A immobilization with zeolitic imidazolate framework for enhancement of ochratoxin A degradation ability. Food Agric. Immunol. 2020, 31, 587-599. [CrossRef] 
32. Dellafiora, L.; Gonaus, C.; Streit, B.; Galaverna, G.; Moll, W.-D.; Vogtentanz, G.; Schatzmayr, G.; Dall'Asta, C.; Prasad, S. An in silico target fishing approach to identify novel ochratoxin a hydrolyzing enzyme. Toxins 2020, 12, 258. [CrossRef]

33. Chang, X.; Wu, Z.; Wu, S.; Dai, Y.; Sun, C. Degradation of ochratoxin A by Bacillus amyloliquefaciens ASAG1. Food Addit. Contam. Part A 2015, 32, 564-571. [CrossRef]

34. Hu, H.; Jia, X.; Wang, Y.; Xiong, L.; Peng, M.; Liang, Z. Detoxification of ochratoxin a by an expressed carboxypeptidase and some isolated peptides from Bacillus subtilis CW14. Toxicon 2019, 158, S67. [CrossRef]

35. Wei, W.; Qian, Y.; Wu, Y.; Chen, Y.; Peng, C.; Luo, M.; Xu, J.; Zhou, Y. Detoxification of ochratoxin A by Lysobacter sp. CW239 and characteristics of a novel degrading gene carboxypeptidase cp4. Environ. Pollut. 2020, 258, 113677. [CrossRef]

36. Petruzzi, L.; Bevilacqua, A.; Baiano, A.; Beneduce, L.; Corbo, M.R.; Sinigaglia, M. In vitro removal of ochratoxin A by two strains of Saccharomyces cerevisiae and their performances under fermentative and stressing conditions. J. Appl. Microbiol. 2014, 116, 60-70. [CrossRef]

37. Ferenczi, S.; Cserháti, M.; Krifaton, C.; Szoboszlay, S.; Kukolya, J.; Szőke, Z.; Kőszegi, B.; Albert, M.; Barna, T.; Mézes, M.; et al. A New Ochratoxin A Biodegradation Strategy Using Cupriavidus basilensis Ör16 Strain. PLoS ONE 2014, 9, e109817. [CrossRef]

38. Fuchs, S.; Sontag, G.; Stidl, R.; Ehrlich, V.; Kundi, M.; Knasmüller, S. Detoxification of patulin and ochratoxin A, two abundant mycotoxins, by lactic acid bacteria. Food Chem. Toxicol. 2008, 46, 1398-1407. [CrossRef]

39. Liuzzi, V.C.; Fanelli, F.; Tristezza, M.; Haidukowski, M.; Picardi, E.; Manzari, C.; Lionetti, C.; Grieco, F.; Logrieco, A.F.; Thon, M.R.; et al. Transcriptional Analysis of Acinetobacter sp. neg1 Capable of Degrading Ochratoxin A. Front. Microbiol. 2017, 7, 2162. [CrossRef]

40. Petchkongkaew, A.; Taillandier, P.; Gasaluck, P.; Lebrihi, A. Isolation of Bacillus spp. from Thai fermented soybean (Thuanao): Screening for aflatoxin B1and ochratoxin A detoxification. J. Appl. Microbiol. 2008, 104, 1495-1502. [CrossRef]

41. Seddi, R.; Chaix, J.-C.; Puigserver, A.; Guo, X.-J. Expression of a soluble and activatable form of bovine procarboxypeptidase A in Escherichia coli. Protein Expr. Purif. 2003, 27, 220-228. [CrossRef]

42. Michaelis, L.; Menten, M.L.; Johnson, K.A.; Goody, R.S. The Original Michaelis Constant: Translation of the 1913 MichaelisMenten Paper. Biochemistry 2011, 50, 8264-8269. [CrossRef]

43. Abrunhosa, L.; Santos, L.; Venâncio, A. Degradation of Ochratoxin A by Proteases and by a Crude Enzyme of Aspergillus niger. Food Biotechnol. 2006, 20, 231-242. [CrossRef]

44. Stoev, S.D. Studies on some feed additives and materials giving partial protection against the suppressive effect of ochratoxin A on egg production of laying hens. Res. Vet. Sci. 2010, 88, 486-491. [CrossRef]

45. Pozzo, L.; Salamano, G.; Mellia, E.; Gennero, M.S.; Doglione, L.; Cavallarin, L.; Tarantola, M.; Forneris, G.; Schiavone, A. Feeding a diet contaminated with ochratoxin A for chickens at the maximum level recommended by the EU for poultry feeds $(0.1 \mathrm{mg} / \mathrm{kg})$. 1. Effects on growth and slaughter performance, haematological and serum traits. J. Anim. Physiol. Anim. Nutr. 2013, 97, 13-22. [CrossRef]

46. Zahoor ul, H.; Khan, M.Z.; Khan, A.; Javed, I. Pathological Responses of White Leghorn Breeder Hens Kept on Ochratoxin A Contaminated Feed. Pak. Vet. J. 2010, 30, 118-123.

47. Wang, W.; Zhai, S.; Xia, Y.; Wang, H.; Ruan, D.; Zhou, T.; Zhu, Y.; Zhang, H.; Zhang, M.; Ye, H.; et al. Ochratoxin A induces liver inflammation: Involvement of intestinal microbiota. Microbiome 2019, 7, 1-14. [CrossRef]

48. Denli, M.; Blandon, J.C.; Guynot, M.E.; Salado, S.; Perez, J.F. Efficacy of a new ochratoxin-binding agent (OcraTox) to counteract the deleterious effects of ochratoxin a in laying hens. Poult. Sci. 2008, 87, 2266-2272. [CrossRef]

49. Armorini, S.; Al-Qudah, K.M.; Altafini, A.; Zaghini, A.; Roncada, P. Biliary ochratoxin A as a biomarker of ochratoxin exposure in laying hens: An experimental study after administration of contaminated diets. Res. Veter Sci. 2015, 100, 265-270. [CrossRef]

50. Giancarlo, B.; Elisabetta, B.; Edmondo, C.; Valeriana, C.; Giuseppina, T. Determination of ochratoxin A in eggs and target tissues of experimentally drugged hens using HPLC-FLD. Food Chem. 2011, 126, 1278-1282. [CrossRef]

51. Piskorska-Pliszczyńska, J.; Juszkiewicz, T. Tissue deposition and passage into eggs of ochratoxin A in Japanese quail. J. Environ. Pathol. Toxicol. Oncol. 1990, 10, 8-10. [PubMed]

52. Gao, C.-Q.; Shi, H.-Q.; Xie, W.-Y.; Zhao, L.-H.; Zhang, J.-Y.; Ji, C.; Ma, Q.-G. Dietary supplementation with acidifiers improves the growth performance, meat quality and intestinal health of broiler chickens. Anim. Nutr. 2021, 7, 762-769. [CrossRef] [PubMed]

53. Yu, Y.; Zhao, F.; Chen, J.; Zou, Y.; Wang, Y.M.; Liu, S.B.; Tan, H.Z. Research Note: Effect of dietary cottonseed meal and soybean oil concentration on digesta passage time and amino acids digestibility in roosters. Poult. Sci. 2021, 100, 101446. [CrossRef] [PubMed]

54. Van der Klis, J.D.; Verstegen, M.; De Wit, W. Absorption of minerals and retention time of dry matter in the gastrointestinal tract of broilers. Poult. Sci. 1990, 69, 2185-2194. [CrossRef]

55. Haridhasapavalan, K.K.; Sundaravadivelu, P.K.; Thummer, R.P. Codon optimization, cloning, expression, purification, and secondary structure determination of human ETS2 Transcription Factor. Mol. Biotechnol. 2020, 62, 485-494. [CrossRef]

56. Sizoo, E.A.; Van Egmond, H.P. Analysis of duplicate 24-hour diet samples for aflatoxin B1, aflatoxin M1and ochratoxin A. Food Addit. Contam. 2005, 22, 163-172. [CrossRef]

57. Hassan, Z.U.; Khan, M.Z.; Khan, A.; Javed, I.; Hussain, Z. Effects of individual and combined administration of ochratoxin A and aflatoxin B1 in tissues and eggs of White Leghorn breeder hens. J. Sci. Food Agric. 2012, 92, 1540-1544. [CrossRef] [PubMed] 